\title{
SUB-BAND ADAPTIVE PRE-EQUALISED OFDM TRANSMISSION
}

\author{
T. Keller, L. Hanzo \\ Dept. of ECS, Univ. of Southampton, SO17 1BJ, UK. \\ Tel: +44-1703-593 125, Fax: +44-1703-594 508 \\ Email:1h@ecs.soton.ac.uk, http://www-mobile.ecs.soton.ac.uk
}

\begin{abstract}
Power-efficient pre--equalisation techniques for subband adaptive OFDM trsnamissions are proposed as an effective means of improving the overall system performance in fading time-dispersive channels. The effects of the time-variant channel are examined and it is shown that adaptive modulation techniques are well suited to pre-equalisation. A range of different throughput and different target bit error rate (BER) systems are designed, which exhibit different amplifier back-off requirements. For example, the $10 \%, 1 \%$ and $0.01 \%$ target BER candidate systems exhibit bits per symbol (BPS) throughputs of about 2,3 and 3.8 around the channel SNR of $15 \mathrm{~dB}$ over the investigated $155 \mathrm{Mbps}, 60 \mathrm{GHz}$ propagation frequency wideband Wireless Asynchronous Transfer Mode (WATM) channel.
\end{abstract}

\section{SYSTEM OVERVIEW}

Sub-band Adaptive Orthogonal Frequency Division Multiplexing (AOFDM) $[1,2]$ has been proposed for duplex communication between two stations over time-variant wideband channels. In order to efficiently react to the time-variant channel quality, the following steps have to be taken:

- Channel quality estimation: In order to appropriately select the transmission parameters to be employed for the next transmission, a reliable prediction of the channel quality during the next active transmit timeslot is necessary.

- Choice of the appropriate parameters for the next transmission: Based on the prediction of the expected channel conditions during the next timeslot, the transmitter has to select the appropriate modulation schemes for the subcarriers.

- Signalling or blind detection of the employed parameters: The receiver has to be informed, as to which set of demodulator parameters to employ for the received packet. This information can either be conveyed within the packet, at the cost of loss of useful data bandwidth, or the receiver can attempt to estimate the parameters employed at the transmitter by means of blind detection mechanisms.

Depending on the channel characteristics, these operations can be performed at either of the duplex stations. If the

VTC'99, AMSTERDAM, NL

Acknowledgement: The financial support of the EPSRC, UK in the framework of the contract GR/K 74043 that of the European Commission and of the Mobile VCE is gratefully acknowledged. channel is reciprocal, then the channel quality estimation for each link can be extracted from the reverse link, and we refer to this regime as open-loop adaptation. In this case, the transmitter has to communicate the transmission parameter set to the receiver, or the receiver can attempt blind detection of the transmission parameters employed [2]. If the channel is not reciprocal, then the channel quality estimation has to be performed at the receiver of the link. In this case, the channel quality measure or the set of requested transmission parameters is communicated to the transmitter in the reverse link. This mode is referred to as closed-loop adaptation.

Equalising the time-dispersive channel's effects in OFDM modems prior to demodulation can be achieved by compensating the received data symbol vector's channel-induced amplitude and phase distortion with the aid of the inverse of the estimated channel's frequency response. Whilst the equalisation at the receiver can ideally reconstruct the amplitude and phase of the received signal, noise enhancement occurs the in subcarriers that have suffered a frequency domain fade during transmission. If the OFDM symbol is pre-distorted with the inverse of the anticipated channel transfer function at the transmitter prior to transmission, then the receiver does not have to perform any frequency-domain equalisation, and therefore avoids the associated noise amplification. The problem associated with "perfect channel inversion" is the prohibitively high output power fluctuation at the transmitter. Adaptive OFDM transmission coupled with pre-equalisation allows us to circumvent these problems. On the basis of the estimated channel transfer function, the adaptive transmit.ter selects a set of modulation modes and power levels for the different subcarriers of the OFDM symbol.

\section{PRE-EQUALISATION AND AOFDM}

\subsection{The Concept}

The estimate of the channel's transfer function can also be employed in duplex modems, in order to dramatically improve the performance of an OFDM system by adapting the subcarrier modulation schemes to the channel transfer function. For subchannels exhibiting a low signal-to-noise ratio (SNR), robust modulation modes can be used, while for subcarriers having a high SNR, high throughput multi-level modulation schemes can be employed $[1,2]$. Additionally, pre-equalisation of the OFDM symbol prior to transmission on the basis of the estimated channel transfer function can be invoked. As discussed above, the received data symbol $R_{n}$ of subcarrier $n$ over a stationary time-dispersive channel can be characterised by:

$$
R_{n}=S_{n} \cdot H_{n}+n_{n}
$$




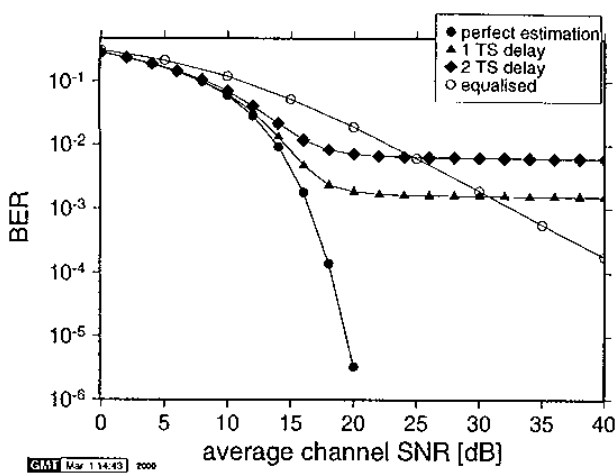

Figure 1: BER performance of the 512-subcarrier 16-QAM OFDM modem over the fading short WATM channel of Figure 2 employing full channel inversion and delays of 0,1 , and 2 timeslots (TS) between the instant of perfect channel estimation and reception. Also depicted is the performance of a perfectly equalised modem under the same channel conditions.

where $S_{n}$ is the transmitted data symbol, $H_{n}$ is the channel transfer function of subcarrier $n$, and $n_{n}$ is a Gaussian noise sample.

Equalisation at the receiver, which is necessary for nondifferential detection of the data symbols, corrects the phase and amplitude of the received data symbols using the estimate of the channel transfer function $\hat{H}_{n}$ as follows:

$$
R_{n}^{\prime}=R_{n} / \hat{H}_{n}=S_{n} \cdot H_{n} / \hat{H}_{n}+n_{n} / \hat{H}_{n} .
$$

If the channel transfer function estimate $\hat{H}_{n}$ is accurate, this operation reconstructs the constellation points before decision. However, the noise sample $n_{n}$ is amplified by the same amount as the signal, therefore failing to improve the SNR of the received sample.

Pre-equalisation for the OFDM modem operates by scaling the data symbol of subcarrier $n, S_{n}$, by a pre-distortion function $E_{n}$, computed from the inverse of the estimated channel transfer function, prior to transmission. At the receiver, no equalisation is performed, hence the received symbols can be expressed as:

$$
R_{n}=S_{n} \cdot E_{n} \cdot H_{n}+n_{n} .
$$

Since no equalisation is performed, there is no noise amplification at the receiver. Similarly to the adaptive modulation techniques alluded to above, pre-equalisation is only applicable to a duplex link, since the transmitted signal is adapted to the specific channel conditions perceived by the receiver. Like for other adaptive schemes, the transmitter needs an estimate of the current frequency-domain channel transfer function, which can be obtained from the received signal in the reverse link.

\subsection{Channel inversion}

The simplest choice of the pre-equalisation transfer function $E_{n}$ is the inverse of the estimated frequency domain channel transfer function, $E_{n}=1 / \hat{H}_{n}$. If the estimation of the channel transfer function is accurate, then channel inversion will result in an AWGN-like channel perceived at the receiver, since all time- and frequency-dependent behaviour of

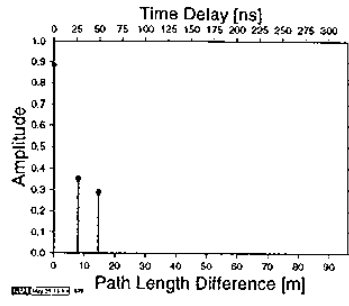

(a) $h(n)$

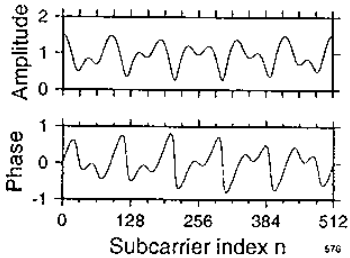

(b) $H_{n}$
Figure 2: Wideband channel (a) - unfaded symbol spaced impulse response $(b)$ - corresponding frequency domain channel transfer function

the channel is cancelled at the transmitter. The Bit Error Rate (BER) performance of such a system, accordingly, is identical to that of the equivalent modem in an AWGN channel. Figure 1 shows the BER performance of a 512 -subcarrier OFDM modem in the Wireless Asynchroneous Transfer Mode (WATM) channel of Figure 2, based upon adjacent up- and downlink Time Division Duplex (TDD) time slots (TS). The TS duration was $2.67 \mu$ s and the channel's Doppler frequency was $2.78 \mathrm{kHz}$, which corresponds to a system employing a carrier frequency of $60 \mathrm{GHz}$, a sampling rate of $225 \mathrm{MSamples} / \mathrm{s}$ and a vehicular velocity of $50 \mathrm{~km} / \mathrm{h}$ or $13 . \overline{8} \mathrm{~m} / \mathrm{s}$.

The average channel SNR on the ordinate axis is the average SNR at the receiver, provided that the pre-equalisation algorithm compensates only for fast fading. The different SNR values can be viewed as a result of additional constant path loss variation, and are not corrected by the preequalisation algorithm. The channel estimation is assumed to be perfect at the time of receiving a timeslot, and this estimation is used for the next reverse link transmission. It can be seen that the performance for 1 timeslot delay between the up- and downlink is fairly close to the Gaussian BER performance for SNR values of up to $10 \mathrm{~dB}$, but that there is a BER floor of about $1.5 \cdot 10^{-3}$. This is due to the delay between the instant of channel estimation and the reception, resulting in a mismatched channel estimate. The curve referred to as "perfect estimation" in the figure represents the case of no delay between channel estimation and reception, which has been implemented by invoking a look-ahead in the channel, so that the transmitter exactly knows the channel transfer function in the future transmit timeslot. In this case, there is no error floor and the system's performance follows closely the theoretical Gaussian curve for 16QAM transmission. A further curve on the graph indicates the measured perfromance of the modem for a delay of 2 timeslots between channel estimation and reception. It can be observed that the BER performance deteriorates further, with a BER floor of $0.7 \%$.

Since the pre-equalisation algorithm amplifies the power in each subcarrier by the corresponding estimate of the channel transfer function, the transmitter's output power fluctuates in harmony with the time--variant channel. The fades in the frequency domain channel transfer function can be deep, hence the transmit power in the corresponding subcarriers may be excessive. Figure 3 shows the histogram of the total OFDM symbol energy at the transmitter's output for the short WLAN channel of Figure 2 with full channel inversion, 


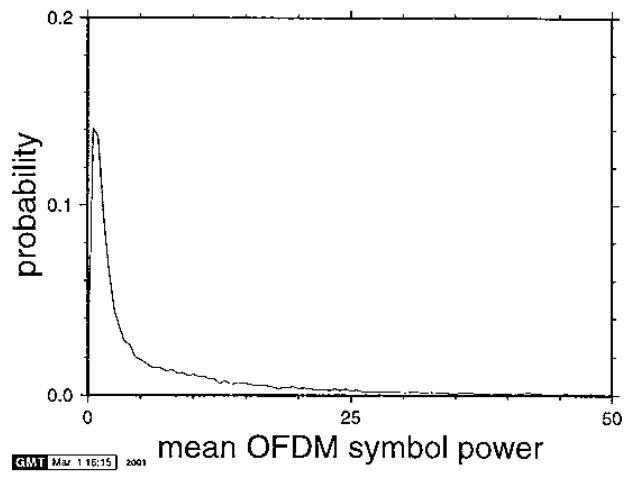

Figure 3: OFDM symbol energy histogram for full channel inversion over the short WATM channel of Figure 2. The corresponding BER curves are given in Figure 1.

normalised to the fixed average output energy. It can be seen that the OFDM symbol energy fluctuates widely, with observed peak values in excess of 55 . The long-term mean symbol energy was measured to be 22.9 , which corresponds to an average output power increase of $13.6 \mathrm{~dB}$. Since this im. poses excessive linearity constraints on the power amplifier, in the next section we considered a range of limited-dynamic range scenarios.

\subsection{Limited dynamic range}

We have seen that although the full channel inversion algorithm produces the best BER performance at the receiver, the output power fluctuations at the transmitter are prohibitive. In order to limit the signal power, the dynamic range of the pre-equalisation algorithm can be limited to a value $l$, so that

$$
\begin{aligned}
& E_{n}=a_{n} \cdot e^{-j \phi_{n}}, \text { with } \\
& \phi_{n}=\angle \hat{H}_{n} \text { and } \\
& a_{n}= \begin{cases}\left|\hat{H}_{n}\right| & \text { for }\left|\hat{H}_{n}\right| \leq l \\
l & \text { otherwise. }\end{cases}
\end{aligned}
$$

Limiting the values of $E_{n}$ to the value of $l$ does not affect the phase of the channel pre-equalisation. Depending on the modulation scheme employed for transmission, reception of the symbols affected by the amplitude limitation is still possible, for example for phase shift keying. Multi-level modulation schemes exploiting the received symbol's amplitude will be affected by the imperfect pre-equalisation. This effect is shown in Figure 4 for 16QAM transmissions and for a maximum power of $l=2$ and $l=4$, which corresponds to a maximal amplitude amplification of $6 \mathrm{~dB}$ and $12 \mathrm{~dB}$, respectively. It can be seen in Figure 4 that for 16QAM transmission, the BER performance curves exhibit BER floors at $2 \%$ and $0.8 \%$ for the $6 \mathrm{~dB}$ and $12 \mathrm{~dB}$ dynamic range limits, respectively. In addition to the time-delay effects highlighted for the full channel inversion algorithm in Figure 1, this is due to the inability of the system to compensate for the channel attenuation in deep fades, which precludes demodulating multi-level symbols correctly. It can be observed that for the higher permissible dynamic range of the pre-equalisation algorithm the BER floor is lower, than for the more limited scenario of $6 \mathrm{~dB}$ clipping, but it is still considerably worse, than that for the full channel inversion. The mean OFDM symbol

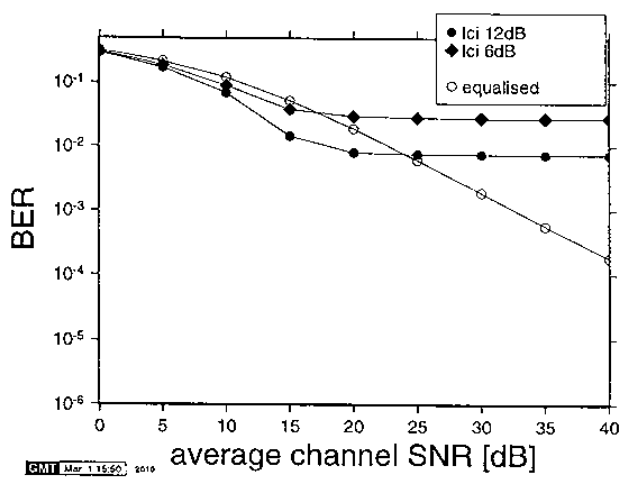

Figure 4: BER performance of the 512-subcarrier 16-QAM OFDM modem over the fading short WATM channel of Figure 2 employing limited channel inversion (lci) and a delay of 1 timeslot between the instants of perfect channel estimation and reception.

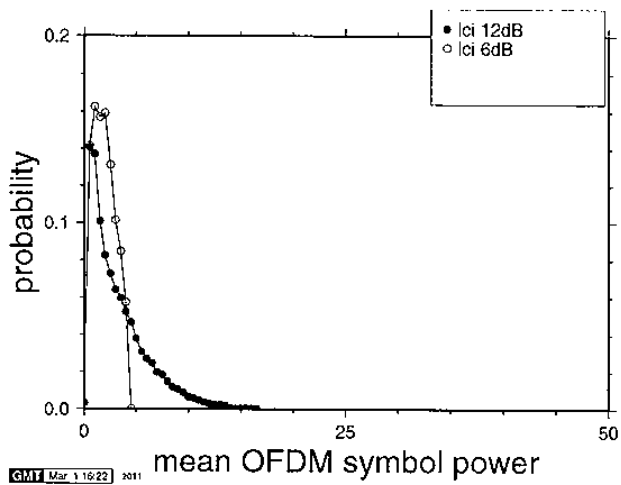

Figure 5: OFDM symbol energy histogram for limited dynamic range channel inversion (lci) over the WATMN channel of Figure 2. The corresponding BER curves are given in Figure 4.

power histogram is shown in Figure 5. Given the maximum allowed amplification factors of 6 and $12 \mathrm{~dB}$, the normalised OFDM symbol power in the figure should be limited to 4 and 16 , respectively. However, higher values are observed, which is due to the OFDM symbol's energy fluctuation as a function of the specific data sequence, if multi-level modulation schemes are used.

\subsection{Pre-equalisation with sub-band blocking}

We have demonstrated in Figures 1 and 4 that while limiting the pre-equalisation function's amplitude can assist in mitigating the problem of transmitter power fluctuation, the imperfect pre-equalisation of the amplitude leads to a BER performance degradation of the system. This BER degradation can be mitigated by identifying the subcarriers that cannot be fully pre-equalised and disabling subsequent transmission in these subcarriers. This "blocking" of the transmission in certain subcarriers can be seen as adaptive modulation with two modulation modes, and introduces the problem of modulation mode signalling. As discussed above, this signalling task 


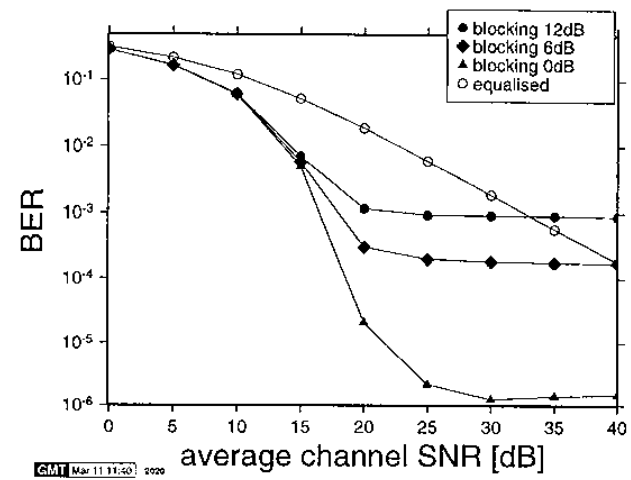

Figure 6: BER performance of the 512-subcarrier 16-QAM OFDM modem over the fading short WATM channel of Figure 2 employing 16 sub-band pre-equalisation with blocking and a delay of 1 timeslot between the instants of perfect channel estimation and reception.

can be solved in different ways, namely by blind detection of blocked subcarriers, or by transmitting explicit signalling information contained in the data block. Employing sub-band adaptivity - rather than subcarrier-by-subcarrier adaptivity - simplifies both the modem mode detection as well as signalling, at the expense of a lower system throughput. In order to keep the system's complexity low and to allow for simple signalling or blind detection, we will assume a 16 sub-band adaptive scheme here.

Analogously to the adaptive modulation schemes alluded to above, the transmitter decides for all subcarriers in each sub-band, whether to transmit data or not. If pre-equalisation is possible under the prevalent maximum power constraints, then the subcarriers are modulated with the pre-equalised data symbols. The information, as to whether a sub-band is used for transmission or not is signalled to the receiver.

Since no attempt is made to transmit in the sub-bands that cannot be pre-equalised, the power not allocated to the blank subcarriers can be used for 'boosting' the databearing sub-bands. This scheme allows for a more flexible pre-equalisation algorithm than the fixed threshold based method described above, and the associated algorithm is summarised as follows:

- Calculate the necessary transmit power $p_{n}$ for each sub-band $n$, assuming perfect pre-equalisation.

- Sort sub-bands according to their required transmit power $p_{n}$

- Select sub-band $n$ with the lowest power $p_{n}$, and add $p_{n}$ to the total transmit power. Repeat this procedure with the next-lowest power, until no further sub-bands can be added without the total power $\sum p_{j}$ exceeding the power limit $l$.

Figure 6 depicts the BER performance over the short WATM channel of Figure 2. It can be seen that the modem performance is improved considerably, when compared to the limited dynamic range algorithm of Figure 4, which can be explained by invoking transmission blocking in the unuseable or low-quality subcarriers. The BER floor stems from the channel's time-variability, as it has been observed for the full channel inversion algorithm in Figure 1. The average throughput figures for the $6 \mathrm{~dB}$ and $12 \mathrm{~dB}$ symbol energy

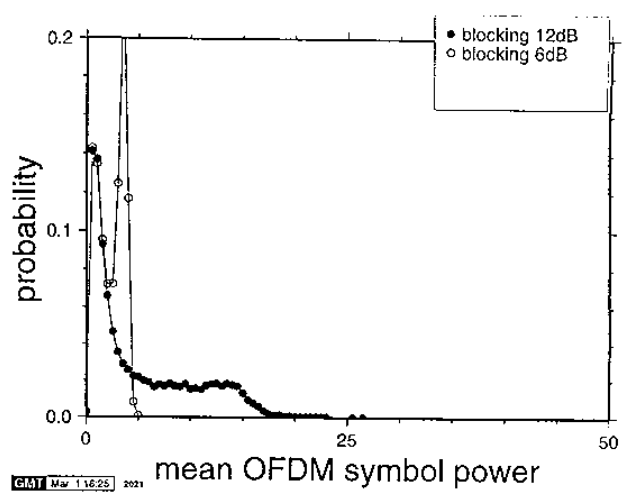

Figure 7: OFDM symbol energy histogram for 16 subband pre-equalisation with subband blocking over the short WATM channel of Figure 2 using 16-QAM. The corresponding BER curves are given in Figure 6

limits are 3.54 and 3.92 bits per data symbol, respectively It can be observed that the BER floor is lower for $l=6 \mathrm{~dB}$ than for $l=12 \mathrm{~dB}$. This is because the effects of the channel variation due to the delay between the instants of channel estimation and reception in the faded subcarriers on the equalisation function is more dramatic, than in the higher-quality subcarriers. The lower the total symbol energy limit $l$, the fewer of the low-quality subcarriers are used for transmission. For both $l=6 \mathrm{~dB}$ and $l=12 \mathrm{~dB}$, the BER performance of the blocking modem is better, than that of the modem employing full channel inversion in Figure 1, provided that 1 time slot delay is assumed. Again, the reason for this is the exclusion of the deeply faded corrupted subcarriers. If the symbol energy is limited to $0 \mathrm{~dB}$, then the BER foor drops to $1.5 \cdot 10^{-6}$ at the expense of the throughput, which attains 2.5 BPS. Figure 7 depicts the mean OFDM symbol energy histogram for this scenario. It can be seen that compared with the limited channel inversion scheme of Figure 5, the allowable symbol energy is more efficiently allocated, with a higher probability of high-energy OFDM symbols. This is the result of the flexible reallocation of energy from blocked subbands, instead of limiting the output power on a subcarrierby-subcarrier basis.

\subsection{Adaptive modulation with spectral pre-distortion}

The pre-equalisation algorithms discussed above invert the channel's anticipated transfer function, in order to transform the resulting channel into a Gaussian-like non-fading channel, whose SNR is dependent only on the path-loss. Sub-band blocking has been introduced above, in order to limit the transmitter's output power, while maintaining the near-constant-SNR across the used subcarriers. The preequalisation algorithms discussed above do not cancel out the channel's path loss, but rely on the receiver's gain control algorithm to automatically account for the channel's average path-loss.

However, if maintaining a constant data throughput is not required by the rest of the communications system, then a fixed BER scheme in conjunction with error correction coding can assist in maximising the system's throughput. The results presented for - what we referred to in [2] as - the target-BER adaptive modulation scheme demonstrated that 


\begin{tabular}{|l|c|c|c|}
\hline target BER & $10^{-4}$ & $1 \%$ & $10 \%$ \\
\hline SNR(BPSK)[dB] & 8.4 & 4.33 & -0.85 \\
SNR(QPSK)[dB] & 11.42 & 7.34 & 2.16 \\
SNR(16QAM)[dB] & 18.23 & 13.91 & 7.91 \\
\hline
\end{tabular}

Table 1: Required target SNR levels for $1 \%$ and $10 \%$ target BER for the different modulation schemes over an AWGN channel.

for the particular turbo coding scheme used an uncoded BER of $1 \%$ resulted in error-free data transmission, and that for an uncoded target BER of $10 \%$ the turbo decoded data BER was below $10^{-5}$. We have seen that it is impossible to exacly reach the anticipated uncoded target BER in conjunction with the adaptive modulation algorithm, since the adaptation algorithm operates in discrete steps between modulation modes.

Combining the target-BER adaptive modulation scheme of [2] and spectral pre-distortion allows the transmitter to react to the channel's time- and frequency-variant nature, in order to fine-tune the behaviour of the adaptive modem in fading channels. It also allows the transmitter to invest the energy that is not used in the disabled or blank sub-bands into the other sub-bands without affecting the equalisation at the receiver.

The combined algorithm for adaptive modulation with spectral pre-distortion described here does not intend to invert the channel's transfer function across the OFDM symbol's range of subcarriers, it is therefore not a pre-equalisation algorithm. Instead, the aim is to transmit a sub-band's data symbols at a specific power level, which ensures a given target SNR at the receiver, that is constant for all subcarriers in the sub-band, which in turn results in the required BER. Clearly, the receiver has to anticipate the different relative power levels for the different modulation schemes, so that error-free demodulation of the multi-level modulation schemes employed can be ensured.

The joint adaptation algorithm requires the estimates of the noise floor level at the receiver as well as the channel transfer function, which includes the path-loss. On the basis of these values, the necessary amplitude of $E_{n}$ required to transmit a data symbol at the subcarrier $n$ for a given received SNR of $\gamma_{n}$ can be calculated as follows:

$$
\left|E_{n}\right|=\frac{\sqrt{N_{0} \cdot \gamma_{n}}}{\left|\hat{H}_{n}\right|}
$$

where $N_{0}$ is the noise floor at the receiver. The phase of $E_{n}$ is used for the pre-equalisation and hence:

$$
\angle E_{n}=-\angle \hat{H}_{n} .
$$

The target SNR, $\gamma_{n}$, of subcarrier $n$, is dependent on the modulation mode of the subcarrier, and determines the system's target BER. We have identified three sets of target SNR values for the modulation schemes, with uncoded target BER values of $1 \%$ and $10 \%$ for use in conjunction with channel coders, as well as a BER of $10^{-4}$ for transmission without channel coding. Table 1 gives an overview of these levels, which have been read from the BER perfromance curves for the different modulation schemes in a Gaussian channel.

Figure 8 shows the performance of the joint pre-distortion and adaptive modulation algorithm in the fading time-dispersive short WATM channel of Figure 2 for the set of different target

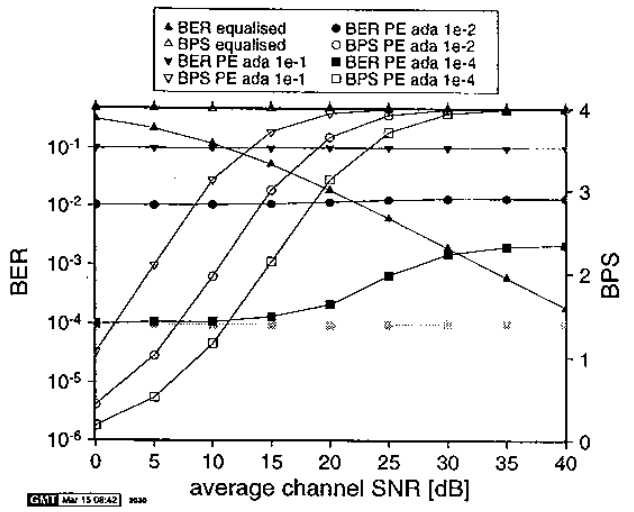

Figure 8: BER performance and throughput of the $16 \mathrm{sub}-$ band adaptive OFDM modem with spectral pre-distortion over the Rayleigh fading time dispersive short WATM channelof Figure 2, and that of the perfectly equalised 16-QAM modem. The grey or 'half-tone' BER curve gives the performance of the adaptive modem for a target BER of $10^{-4}$ with no delay between channel estimation and transmission, while the other results assume 1 timeslot delay between up- and downlink.

BER values of Table 1, as well as the comparison curves of the perfectly equalised 16-QAM modem under the same channel conditions. It can be seen that the actual BER achieved by the system is close to the BER targets. Specifically, for a target BER of $10 \%$, no perceptible deviation from the target has been recorded, while for the lower BER targets the deviations increase for higher channel SNRs. For a target BER of $1 \%$, the highest measured deviation is at the SNR of $40 \mathrm{~dB}$, where the recorded BER is $1.36 \%$. For the target BER of $10^{-4}$, the BER deviation is small at OdB SNR, but at an SNR of $40 \mathrm{~dB}$, the experimental BER is $2.2 \cdot 10^{-3}$. This increase of the BER with increasing SNR is due to the rapid channel variations in the deeply faded subcarriers, which are increasingly used at higher SNR values. The grey or 'half-tone' curve in the figure denotes the system's performance, if no delay is present between the channel estimation and the transmission. In this case, the simulated BER shows only very little deviation from the target BER value. This is consistent with the behaviour of the full channel inversion pre-equalising modem.

In conclusion, the combination of limited channel inversion and adaptive modulation based OFDM is a promising candidate for further study, which offers further performance improvements over that due to AOFDM in isolation. For example, the proposed $10 \%, 1 \%$ and $0.01 \%$ target BER candidate systems exhibit bits per symbol (BPS) throughputs of about 2, 3 and 3.8 around the channel SNR of $15 \mathrm{~dB}$ over the investigated $155 \mathrm{Mbps}, 60 \mathrm{GHz}$ propagation frequency wideband WATM channel of Figure 2.

\section{REFERENCES}

[1] T. Keller and L. Hanzo, "Adaptive orthogonal frequency division multiplexing schemes," in Proc. ACTS Summit, (Rhodos, Greece), pp. 794-799, June 1998.

[2] T. Keller, L. Hanzo: Blind-detection Assisted Subband Adaptive Turbo-Coded OFDM Schemes, Proc. of VTC'99, Houston, USA, May, 1999, pp 489-493 